\title{
Improved Quasiuniform Stability for Fractional Order Neural Nets with Mixed Delay
}

\author{
Omar Naifar $\mathbb{D}^{1}{ }^{1}$ Assaad Jmal, ${ }^{1}$ A. M. Nagy $\mathbb{D}^{2,3}$ and Abdellatif Ben Makhlouf ${ }^{4,5}$ \\ ${ }^{1}$ Control and Energy Management Laboratory, National School of Engineering, Sfax University, BP 1173, Sfax 3038, Tunisia \\ ${ }^{2}$ Department of Mathematics, Faculty of Science, Benha University, 13518 Benha, Egypt \\ ${ }^{3}$ Kuwait University, Safat, Kuwait \\ ${ }^{4}$ Jouf University, Aljouf, Saudi Arabia \\ ${ }^{5}$ University of Sfax, Sciences Faculty, Mathematics Department, Sfax, Tunisia
}

Correspondence should be addressed to Omar Naifar; email.naifar@gmail.com

Received 30 August 2020; Revised 2 November 2020; Accepted 11 November 2020; Published 29 November 2020

Academic Editor: Mariusz Michta

Copyright ( $\odot 2020$ Omar Naifar et al. This is an open access article distributed under the Creative Commons Attribution License, which permits unrestricted use, distribution, and reproduction in any medium, provided the original work is properly cited.

In the present paper, a quasiuniform stability result for fractional order neural networks with mixed delay is developed, based on the generalized Gronwall inequality and the Caputo fractional derivative. Sufficient conditions are derived to ensure the quasiuniform stability of the considered neural nets system. A clarification example is carried out not only to validate the authors' theoretical results but also to show the superiority of the developed work (in terms of improved stability), compared with other similar works already published in the literature.

\section{Introduction}

A fractional order system is a dynamical system that can be modeled by fractional differential equations, carried with a noninteger derivative [1]. Such systems are said to have fractional dynamics. Integrals and derivatives of fractional orders are used to illustrate objects that can be described by power-law nonlocality [2] or power-law long-range dependence or fractal properties. Note that the fractional-order calculus has been used in studying the systems' dynamics in many fields such as electrochemistry, physics, viscoelasticity, biology, and chaotic systems [1]. In a related context, the evolution of science and engineering systems has considerably stimulated the employment of the fractional calculus in many areas of the control theory, in the last decades, and this includes stability [3-6], finite-time stability (FTS) [7-9], stabilization [10], observer design [10, 11], and fault estimation [12-14].

Currently, the analysis of stability for fractional order neural network systems has received some attention, and some results have been published in this context. For example, in [9], the authors have presented an original scheme, in order to study the finite-time stability of the equilibrium point and to prove its existence and uniqueness, for Caputo-Katugampola fractional-order neural networks, with time delay. The proposed scheme, therein, has used a newly introduced fractional derivative concept in the literature, which is the Caputo-Katugampola fractional derivative. In another relevant work [15], the authors have studied the quasiuniform stability of Caputo-type fractional-order neural networks with mixed delay. In [16], the authors have investigated the impulsive effects on stability and passivity analysis of memristor-based fractional order competitive neural networks. In [17], the idea was to study the global Mittag-Leffler stability and stabilization of fractional order quaternion-valued memristive neural networks. In a related context, another research work [18] has focused on the hybrid control problem for projective lag synchronization of Riemann-Liouville fractional order memristive BAM neural networks with mixed delays.

On another hand, note that time delays are so frequent in the electronic implementation of neural networks. This is why it is of great importance to consider these delays in studying the stability of neural nets. So far, various works have been developed by researchers, on this issue. For instance, in [19], the authors have developed an asymptotic 
stability analysis for discrete-time stochastic quaternionvalued neural networks with time delays. In [20], the authors have considered a delay-dividing approach to robust stability investigation of uncertain stochastic complex-valued Hopfield delayed neural networks. In another work [21], the idea was to study the robust stability of complex-valued stochastic neural networks with time-varying delays and parameter uncertainties.

Motivated by the above discussion, the present paper aims to present an improved quasiuniform stability result for fractional order neural networks with mixed delay, using the generalized Gronwall inequality. Establishing sufficient criteria for the resolution of such a problem is quite important and challenging. It is important to mention that the present work is a generalization of [7]. Finally, and to show the superiority of the present developed work (in terms of improved stability), the same numerical example as in [15] is studied, and it is shown that our theoretical results are more accurate than those presented in [15].

As a summary, here are the advantages of the proposed work, compared to other existing literature works:

(i) Compared to [15], where exactly the same problem is considered for the same class of neural networks, the present paper presents an improved quasiuniform stability result (see Section 5, for comparison between both papers)

(ii) Compared to $[16,17]$, where time-delay is not considered, the present paper presents the advantage of taking delay into consideration

(iii) Compared to [9], where the authors have studied the finite-time stability for Caputo-Katugampola fractional order delayed neural networks, the present paper present the advantage of considering mixed delay (with two delay parameters), not only one delay parameter (as in [9])

(iv) Compared to [19-21], the present paper presents the advantage of considering a fractional order model of neural networks, which is more general of integerorder models
The rest of the paper is organized as follows. Some necessary definitions and lemmas are given in Section 2. In Section 3, the fractional-order neural network model is introduced. Sufficient conditions ensuring the quasiuniform stability of the fractional order neural nets with mixed delay are presented in Section 4. Finally, a numerical example is investigated in Section 5.

\section{Preliminaries}

In this section, some definitions and results related to the fractional calculus are presented. The literature contains different definitions of the fractional derivative $[22,23]$. In this paper, the Caputo definition is adopted.

Definition 1. Given $0<\alpha<1$, the Caputo fractional derivative is defined as

$$
{ }^{C} D_{t_{0}, t}^{\alpha} x(t)=\frac{1}{\Gamma(1-\alpha)} \frac{\mathrm{d}}{\mathrm{d} t} \int_{t_{0}}^{t}(t-s)^{-\alpha}\left(x(s)-x\left(t_{0}\right)\right) \mathrm{d} s .
$$

There exists a frequently used function in the solution of fractional order systems, named the Mittag-Leffler function. This function is a generalization of the exponential one. In this context, the following definition is given.

Definition 2. The Mittag-Leffler function with two parameters is defined as

$$
E_{\alpha, \beta}(z)=\sum_{k=0}^{+\infty} \frac{z^{k}}{\Gamma(k \alpha+\beta)},
$$

where $\alpha>0, \quad \beta>0$, and $z \in \mathbb{C}$. For $\beta=1$, one has $E_{\alpha}(z)=E_{\alpha, 1}(z)$. Furthermore, $E_{1,1}(z)=e^{z}$.

Lemma 1 (see [24]). Let $a(t), b(t), g(t)$, and $u(t)$ be nonnegative functions on $I=[0, T), \quad T \leq+\infty, \alpha \in(0,1)$. Moreover, if are locally summable on I. If $b(t)$ and $a(t)$ are nondecreasing continuous functions on I bounded by a constant $>0$, let $p \geq q>0$ and $\lambda>0$ such that

$$
u^{p}(t) \leq a(t)+b(t) \int_{0}^{t} u^{q}(s) \mathrm{d} s+g(t) \int_{0}^{t}(t-s)^{\alpha-1} u^{q}(s) \mathrm{d} s, \quad t \in[0, T)
$$

then we have the following explicitly bound for $u$

$$
u(t) \leq\left\{\hat{a}(t)+\sum_{k=1}^{\infty} \sum_{i=0}^{k} \frac{\left(\begin{array}{l}
k \\
i
\end{array}\right)\left[q \lambda^{((q-p) / p)}\right]^{k}[b(t)]^{k-i}[\Gamma(\alpha) g(t)]^{i}}{p^{k} \Gamma(i \alpha+k-i)} \int_{0}^{t}(t-s)^{i \alpha-i-1+k} \widehat{a}(s) \mathrm{d} s\right\}^{1 / p}, \quad t \in[0, T),
$$


provided that

In addition, if $a(t)$ is nondecreasing on $[0, T)[0, T)$, then

$$
\widehat{a}(t):=a(t)+\frac{(p-q) \lambda^{q / p}\left[\alpha t b(t)+t^{\alpha} g(t)\right]}{\alpha p} .
$$

$$
u(t) \leq \sqrt[p]{\widehat{a}(t) E_{\alpha}\left(\frac{q \Gamma(\alpha) g(t) \lambda^{((q-p) / p)} t^{\alpha}}{p}\right) \exp \left(\frac{q t \lambda^{((q-p) / p)} b(t)}{p \alpha}\right)}, \quad t \in[0, T) .
$$

\section{Problem Statement}

The dynamic behavior of a continuous fractional order neural network with mixed delay can be described by the following differential equation:

$$
\begin{aligned}
\left\{{ }^{C} D_{t_{0}, t}^{\alpha} x_{i}(t)=\right. & -c_{i} x_{i}(t)+\sum a_{i j} f_{j}\left(x_{j}(t)\right)+\sum b_{i j} g_{j}\left(x_{j}(t-\tau)\right) \\
& +\sum m_{i j} \int_{t-\sigma}^{t} h_{i}\left(x_{i}(u)\right) \mathrm{d} u+I_{i}(t), x_{i}(t)=\psi_{i}(t), \quad t \in[-\gamma, 0), \gamma \in \max \{\tau, \sigma\}
\end{aligned}
$$

or equivalently

$$
\left\{{ }^{C} D_{t_{0}, t}^{\alpha} x(t)=-A_{0} x(t)+A_{1} F(x(t))+\mathrm{BG}(x(t-\tau))+M \int_{t-\sigma}^{t} H(x(u)) \mathrm{d} u+I(t), x(t)=\psi(t), \quad t \in[-\gamma, 0), \gamma \in \max \{\tau, \sigma\},\right\}
$$

where $0<\alpha<1 j=1,2, \ldots, n, n$ corresponds to the number of units in a neural network, $x(t)=$ $\left(x_{1}(t), x_{2}(t), \ldots, x_{n}(t)\right)^{T}$ corresponds to the state vector at time $t, F(x(t))=\left(f_{1}\left(x_{1}(t)\right), f_{2}\left(x_{2}(t)\right), \ldots, f_{n}\left(x_{n}(t)\right)\right)^{T}$, $H(x(t))=\left(h_{1}\left(x_{1}(t)\right), h_{2}\left(x_{2}(t)\right), \ldots, h_{n}\left(x_{n}(t)\right)\right)^{T}$ denotes the activation function of the neurons, $A_{0}=\operatorname{diag}\left(c_{i}>0\right)$ $A_{1}=\left(a_{i j}\right), B=\left(b_{i j}\right)$, and $M=\left(m_{i j}\right)$ are constant matrices; $c_{i}$ denotes the rate with which the $i$ th unit will reset its potential to the resting state in isolation when disconnected from the network. $A_{1}=\left(a_{i j}\right), B=\left(b_{i j}\right)$ and $M=\left(m_{i j}\right)$ refer to the connection of the $j$ th neuron to the $i$ th neuron at time $t, t-\tau$ and $t-\sigma$, respectively, where $\tau$ and $\sigma$ are two parameters representing time-delay. $I=\left(I_{1}(t), I_{2}(t), \ldots, I_{n}(t)\right)^{T}$ is an external bias vector.

Remark 1. For the initial conditions associated with system (7), it is usually assumed that $\psi_{i}(s) \in C([-\gamma, 0], R)$. Denote $\|\psi\|=\sup _{s \in[-\gamma, 0]}\|\psi(s)\|$.

Suppose that $x(t)$ and $y(t)$ are any two solutions of (8) with different initial functions $\psi \in C$ and $\phi \in C$, $\psi(0)=\phi(0)=0$. Let $x(t)-y(t)=e(t)=\left(e_{1}(t), e_{2}(t), \ldots\right.$, $\left.e_{n}(t)\right)^{T}, \varphi=\psi-\phi$, then one obtains the error system:

$$
\begin{aligned}
& \left\{{ }^{C} D_{t_{0}, t}^{\alpha} e(t)=-A_{0} e(t)+A_{1}(F(x(t))-F(y(t)))+B(G(x(t-\tau))\right. \\
& \left.\quad-G(y(t-\tau)))+M \int_{t-\sigma}^{t}(H(x(u))-H(y(u))) \mathrm{d} u, e(t)=\varphi(t), \quad t \in[-\gamma, 0), \gamma \in \max \{\tau, \sigma\},\right\}
\end{aligned}
$$

where $\varphi \in C, \varphi(0)=0$ is the initial function of system (8); we define the norm $\|\varphi\|=\sup _{s \in[-\gamma, 0]}\|, \varphi(s)\|$.
Definition 3 (see [15]). System given by (8), satisfying initial condition $e(t)=\varphi(t),-\tau \leq t \leq 0$, is quasiuniformly stable, 
with respect to $\{\delta, \varepsilon, T\}, \delta<\varepsilon$, if and only if $\|\varphi\|<\delta$ implies $\|e(t)\|<\varepsilon, \forall t \in[0, T]$.

Remark 2. The considered model (8) refers to fractional order artificial neural networks (ANN), with mixed delay. ANN represent a growing used tool in modern technology. And time-delays should be considered in studying such systems because these delays are frequent in electronic implementation of ANN. The importance of this manuscript consists on the fact that it is crucial to check if the designed ANN represent stable dynamics or not. And this clarifies the motivation behind the present study of quasiuniform stability for these ANN.

The following assumptions are to be made:

(1) Denote $\quad\|x\|=\sum_{i=1}^{n}\left|x_{i}\right| \quad$ and $\left\|A_{1}\right\|=\max _{1 \leq j \leq n} \sum_{i=1}^{n}\left|a_{i j}\right|$, which are the Euclidean vector norm and matrix norm, respectively; $x_{i}$ and $a_{i j}$ are the elements of the vector $x$ and the matrix $A_{1}$, respectively. Let $a_{0}=\left\|A_{0}\right\|, a_{1}=\left\|A_{1}\right\|, b=\|B\|$ and $m_{1}=\|M\|$.

(2) The neuron activation functions $F(x), G(x)$, and $H(x)$ are Lipschitz continuous, namely, there exist positive constants $k_{F}, k_{G}$, and $k_{H}$ such that

$$
\begin{aligned}
&\|F(u)-F(v)\| \leq k_{F}\|u-v\|, \\
&\|G(u)-G(v)\| \leq k_{G}\|u-v\|, \\
&\|H(u)-H(v)\| \leq k_{H}\|u-v\|, \\
& \forall u, v \in \mathbb{R}^{n} .
\end{aligned}
$$

The integral equation corresponding to (9) is given by

$$
e(t)=\varphi(0)+\frac{1}{\Gamma(\alpha)} \int_{0}^{t}(t-s)^{\alpha-1}\left[\begin{array}{c}
-A_{0} e(s)+A_{1}(F(x(s))-F(y(s)))+B(G(x(s-\tau))-G(y(s-\tau))) \\
+M \int_{s-\sigma}^{s}(H(x(u))-H(y(u))) \mathrm{d} u
\end{array}\right] \mathrm{d} s .
$$

Let $\quad T>\gamma \quad$ and $\quad I=[0, T]=\cup_{k=0}^{m}[k \gamma,(k+1) \gamma] \cup$ $[(m+1) \gamma, T]$, with $(m+1) \gamma<T \leq(m+2) \gamma$

$$
r_{T}(\gamma) E_{\alpha}\left(v_{3} T^{\alpha}\right) e^{\left(v_{2} T^{\alpha+1}\right) /(\alpha \Gamma(\alpha+1))} \leq \frac{\varepsilon}{\delta},
$$

\section{Main Result}

Theorem 1. System (9) is quasiuniformly stable with respect to $\{\delta, \varepsilon, T\}(\delta<\varepsilon)$, if the following condition is satisfied:

$$
\begin{aligned}
r_{T}(\gamma) & =\left[1+\left(\frac{v_{0}+v_{1} r_{m+1}(\gamma) E_{\alpha}\left(v_{3}((m+1) \gamma)^{\alpha}\right) e^{\left(v_{2}((m+1) \gamma)^{\alpha+1}\right) /(\alpha \Gamma(\alpha+1))}}{\Gamma(\alpha+1)}\right) T^{\alpha}\right], \\
r_{k+1}(\gamma) & =\left[1+\left(\frac{v_{0}+v_{1} r_{k}(\gamma) E_{\alpha}\left(v_{3}(k \gamma)^{\alpha}\right) e^{\left(\left(v_{2}(k \gamma)^{\alpha+1}\right) / \alpha \Gamma(\alpha+1)\right)}}{\Gamma(\alpha+1)}\right)((k+1) \gamma)^{\alpha}\right], \quad 0 \leq k \leq m, \\
r_{0}(\gamma) & =1, \\
v_{0} & =\|M\| k_{H} \sigma, \\
v_{1} & =\|B\| k_{G}, \\
v_{2} & =\|M\| k_{H}, \\
v_{3} & =\left\|A_{0}\right\|+\left\|A_{1}\right\| k_{F} .
\end{aligned}
$$


Mathematical Problems in Engineering

5

Proof. We have, for $t \in[0, T] t \in[0, T]$,

$$
\begin{aligned}
\|e(t)\| \leq & \|\varphi(0)\|+\frac{1}{\Gamma(\alpha)} \int_{0}^{t}(t-s)^{\alpha-1}\left[\left\|A_{0}\right\|\|e(s)\|+\left\|A_{1}\right\| k_{F}\|e(s)\|+\|B\| k_{G}\|e(s-\tau)\|+\|M\| k_{H} \int_{s-\sigma}^{s}\|e(u)\| \mathrm{d} u\right] \mathrm{d} s \\
\leq & \|\varphi(0)\|+\frac{1}{\Gamma(\alpha)} \int_{0}^{t}(t-s)^{\alpha-1}\left(\left\|A_{0}\right\|+\left\|A_{1}\right\| k_{F}\right)\|e(s)\| \mathrm{d} s+\frac{1}{\Gamma(\alpha)} \int_{0}^{t}(t-s)^{\alpha-1}\|B\| k_{G}\|e(s-\tau)\| \mathrm{d} s \\
& +\frac{1}{\Gamma(\alpha)} \int_{0}^{t}(t-s)^{\alpha-1}\|M\| k_{H} \int_{-\sigma}^{t}\|e(u)\| \mathrm{d} u \mathrm{~d} s \\
\leq & \|\varphi\|+\frac{1}{\Gamma(\alpha)} \int_{0}^{t}(t-s)^{\alpha-1}\left(\left\|A_{0}\right\|+\left\|A_{1}\right\| k_{F}\right)\|e(s)\| \mathrm{d} s+\frac{1}{\Gamma(\alpha)} \int_{0}^{t}(t-s)^{\alpha-1}\|B\| k_{G}\|e(s-\tau)\| \mathrm{d} s \\
& +\frac{1}{\Gamma(\alpha)}\|M\| k_{H} \int_{-\sigma}^{t}\|e(u)\| \mathrm{d} u \int_{0}^{t}(t-s)^{\alpha-1} \mathrm{~d} s \\
\leq & \|\varphi\| \frac{\|M\| k_{H} \sigma t^{\alpha}\|\varphi\|}{\Gamma(\alpha+1)}+\frac{\|B\| k_{G}}{\Gamma(\alpha)} \int_{0}^{t}(t-s)^{\alpha-1}\|e(s-\tau)\| \mathrm{d} s+\frac{\|M\| k_{H} t^{\alpha}}{\Gamma(\alpha+1)} \int_{0}^{t}\|e(s)\| \mathrm{d} s+\frac{\left\|A_{0}\right\|+\left\|A_{1}\right\| k_{F}}{\Gamma(\alpha)} \int_{0}^{t}(t-s)^{\alpha-1}\|e(s)\| \mathrm{d} s \\
\leq & \left(1+\frac{v_{0}}{\Gamma(\alpha+1)} t^{\alpha}\right)\|\varphi\|+\frac{v_{1}}{\Gamma(\alpha)} \int_{0}^{t}(t-s)^{\alpha-1}\|e(s-\tau)\| \mathrm{d} s+\frac{v_{2}}{\Gamma(\alpha+1)} t^{\alpha} \int_{0}^{t}\|e(s)\| \mathrm{d} s+\frac{v_{3}}{\Gamma(\alpha)} \int_{0}^{t}(t-s)^{\alpha-1}\|e(s)\| \mathrm{d} s
\end{aligned}
$$

For $t \in[0, \gamma]$, we have

$$
\begin{aligned}
\|e(t)\| & \leq\left(1+\frac{v_{0}}{\Gamma(\alpha+1)} t^{\alpha}\right)\|\varphi\|+\frac{v_{1}}{\Gamma(\alpha+1)} t^{\alpha}\|\varphi\|+\frac{v_{2}}{\Gamma(\alpha+1)} t^{\alpha} \int_{0}^{t}\|e(s)\| \mathrm{d} s+\frac{v_{3}}{\Gamma(\alpha)} \int_{0}^{t}(t-s)^{\alpha-1}\|e(s)\| \mathrm{d} s \\
& \leq\left(1+\frac{\left(v_{0}+v_{1}\right)}{\Gamma(\alpha+1)} \gamma^{\alpha}\right)+\frac{v_{2}}{\Gamma(\alpha+1)} \gamma^{\alpha} \int_{0}^{t}\|e(s)\| \mathrm{d} s+\frac{v_{3}}{\Gamma(\alpha)} \int_{0}^{t}(t-s)^{\alpha-1}\|e(s)\| \mathrm{d} s .
\end{aligned}
$$

Using Lemma 1 , for $t \in[0, \gamma]$, one gets

$$
\begin{aligned}
\|e(t)\| & \leq\left(1+\frac{\left(v_{0}+v_{1}\right)}{\Gamma(\alpha+1)} \gamma^{\alpha}\right) E_{\alpha}\left(v_{3} t^{\alpha}\right) e^{\left(\left(v_{2} \gamma^{\alpha}\right) / \alpha \Gamma(\alpha+1)\right) t}\|\varphi\| \\
& \leq r_{1}(\gamma) E_{\alpha}\left(v_{3} \gamma^{\alpha}\right) e^{\left(\left(v_{2} \gamma^{\alpha+1}\right) / \alpha \Gamma(\alpha+1)\right)}\|\varphi\| .
\end{aligned}
$$

For $t \in[0,2 \gamma]$, one gets

$$
\begin{aligned}
\|e(t)\| \leq & \left(1+\frac{v_{0}}{\Gamma(\alpha+1)} t^{\alpha}\right)\|\varphi\|+\frac{v_{1}}{\Gamma(\alpha+1)} t^{\alpha} r_{1}(\gamma) E_{\alpha}\left(v_{3} \gamma^{\alpha}\right) e^{\left(\left(v_{2} \gamma^{\alpha+1}\right) /(\alpha \Gamma(\alpha+1))\right)}\|\varphi\|+\frac{v_{2}}{\Gamma(\alpha+1)} t^{\alpha} \int_{0}^{t}\|e(s)\| \mathrm{d} s \\
& +\frac{v_{3}}{\Gamma(\alpha)} \int_{0}^{t}(t-s)^{\alpha-1}\|e(s)\| \mathrm{d} s \\
\leq & {\left[1+\left(\frac{v_{0}+v_{1} r_{1}(\gamma) E_{\alpha}\left(v_{3} \gamma^{\alpha}\right) e^{\left(\left(v_{2} \gamma^{\alpha+1}\right) /(\alpha \Gamma(\alpha+1))\right)}}{\Gamma(\alpha+1)}\right)(2 \gamma)^{\alpha}\right]\|\varphi\|+\frac{v_{2}}{\Gamma(\alpha+1)}(2 \gamma)^{\alpha} \int_{0}^{t}\|e(s)\| \mathrm{d} s+\frac{v_{3}}{\Gamma(\alpha)} \int_{0}^{t}(t-s)^{\alpha-1}\|e(s)\| \mathrm{d} s . }
\end{aligned}
$$


Using Lemma 1 , for $t \in[0,2 \gamma]$, we get

$$
\begin{aligned}
\|e(t)\| & \leq\left[1+\left(\frac{v_{0}+v_{1} r_{1}(\gamma) E_{\alpha}\left(v_{3} \gamma^{\alpha}\right) e^{\left(\left(v_{2} \gamma^{\alpha+1}\right) /(\alpha \Gamma(\alpha+1))\right)}}{\Gamma(\alpha+1)}\right)(2 \gamma)^{\alpha}\right] E_{\alpha}\left(v_{3} t^{\alpha}\right) e^{\left(\left(v_{2}(2 \gamma)^{\alpha}\right) /(\alpha \Gamma(\alpha+1))\right) t}\|\varphi\| \\
& \leq r_{2}(\gamma) E_{\alpha}\left(v_{3}(2 \gamma)^{\alpha}\right) e^{\left(\left(v_{2}(2 \gamma)^{\alpha+1}\right) /(\alpha \Gamma(\alpha+1))\right)}\|\varphi\| .
\end{aligned}
$$

Using the same approach, one gets, for $t \in[0,(k+1) \gamma]$ $\|e(t)\| \leq r_{k+1}(\gamma) E_{\alpha}\left(v_{3}((k+1) \gamma)^{\alpha}\right) e^{\left(\left(v_{2}((k+1) \gamma)^{\alpha+1}\right) /(\alpha \Gamma(\alpha+1))\right)}\|\varphi\|$.

Finally, for $t \in[0, T]$, one gets

$$
\|e(t)\| \leq r_{T}(\gamma) E_{\alpha}\left(v_{3} T^{\alpha}\right) e^{\left(\left(v_{2} T^{\alpha+1}\right) /(\alpha \Gamma(\alpha+1))\right)}\|\varphi\| .
$$

Remark 3. Note that $r_{0}(\gamma) \leq r_{1}(\gamma) \leq \cdots \leq r_{T}(\gamma)$.

Remark 4. If $0<T \leq \gamma$, then we can obtain the finite-time stability for system (9) under condition (12), where

$$
r_{T}(\gamma)=\left[1+\left(\frac{v_{0}+v_{1}}{\Gamma(\alpha+1)}\right) T^{\alpha}\right] .
$$

\section{Clarification Example}

An illustrative example is described to further show the theoretical result. The goal through this section is to show that the quasiuniform stability, obtained with our computations, is better than the one obtained in [15]. That is to say, the authors here aim to compute the value of $T$ and to compare it to the one found in [15], rather than to give the figures of the solutions $x_{1}(t)$ and $x_{2}(t)$. As for figures, the readers can have an idea about them by returning to [15].

Consider the two-state fractional order mixed delay neural network model as in [15]:

$$
\begin{aligned}
{ }^{C} D_{t_{0}, t}^{\alpha}\left(x_{1}(t)\right)= & -0.1 x_{1}(t)+0.2 f_{1}\left(x_{1}(t)\right)-0.1 f_{2}\left(x_{2}(t)\right)-0.5 g_{1}\left(x_{1}(t-\tau)\right)-0.1 g_{2}\left(x_{2}(t-\tau)\right) \\
& +\int_{t-\sigma}^{t}\left[0.4 h_{1}\left(x_{1}(u)\right)-0.1 h_{2}\left(x_{2}(u)\right)\right] \mathrm{d} u, \\
{ }^{C} D_{t_{0}, t}^{\alpha}\left(x_{2}(t)\right)= & -0.1 x_{2}(t)+0.1 f_{1}\left(x_{1}(t)\right)-0.2 f_{2}\left(x_{2}(t)\right)-0.2 g_{1}\left(x_{1}(t-\tau)\right)-0.1 g_{2}\left(x_{2}(t-\tau)\right) \\
& +\int_{t-\sigma}^{t}\left[0.1 h_{1}\left(x_{1}(u)\right)+0.2 h_{2}\left(x_{2}(u)\right)\right] \mathrm{d} u,
\end{aligned}
$$

with constant delays $\tau=0.2$ and $\sigma=0.05$, and the activation function considered by $f_{i}\left(x_{i}(t)\right)=g_{i}\left(x_{i}(t)\right)=h_{i}\left(x_{i}(t)\right)=$ $\tanh (x)(i=1,2)$,. So $k_{F}=k_{G}=k_{H}=1$ and

$$
\begin{aligned}
A_{0} & =\left(\begin{array}{cc}
0.1 & 0 \\
0 & 0.1
\end{array}\right), \\
A_{1} & =\left(\begin{array}{cc}
0.2 & -0.1 \\
0.1 & -0.2
\end{array}\right), \\
B & =\left(\begin{array}{cc}
-0.5 & -0.1 \\
-0.2 & -0.1
\end{array}\right), \\
M & =\left(\begin{array}{cc}
0.4 & -0.1 \\
0.1 & 0.2
\end{array}\right),
\end{aligned}
$$

and then we have $a_{0}=0.1, a_{1}=0.3, b=0.7$, and $m_{1}=0.5$.
The goal is to solve inequality (12) with $\delta=0.1, \varepsilon=1$, for various values of $\alpha$ and compare with the results obtained in [15]. After solving inequality (12), one can see that Theorem 1 guarantees the quasiuniform stability. If we take $\alpha=0.7$, then $\alpha=0.3 \alpha=0.3$. Theorem 1 provides the values $T=0.7$ and $T=0.3$, while the results obtained in [15] are $T=0.6699$ and $T=0.0522$, respectively. It is obvious that values of $T$ computed by our inequality given in (12) are larger than those obtained in [15], and this example shows that our theoretical results are more accurate than those presented in [15].

\section{Conclusion}

In this paper, a new quasiuniform stability result for fractional order neural networks with a mixed delay has been proposed. The developed scheme is based on the generalized Gronwall inequality. An illustrative example shows that the quasiuniform stability criterion derived in this paper 
provides better results than the ones found in a similar existing literature article [15].

As for possible related future work, the authors intend to extend the present results to fractional order neural networks with time-varying delays, instead of constant delays.

\section{Data Availability}

It is a purely mathematical research work, which is based on theoretical analysis and developing theorem proofs.

\section{Conflicts of Interest}

The authors declare that there are no conflicts of interest..

\section{References}

[1] C. A. Monje, Y. Chen, B. M. Vinagre, D. Xue, and V. FeliuBatlle, Fractional Order Systems and Controls: Fundamentals and Applications, Springer, Berlin, Germany, 2010.

[2] C. Cattani, H. M. Srivastava, and X. J. Yang, Fractional Dynamics, Walter de Gruyter KG, Berlin, Germany, 2015.

[3] O. Naifar, A. B. Makhlouf, and M. A. Hammami, "Comments on "Lyapunov stability theorem about fractional system without and with delay"' Communications in Nonlinear Science and Numerical Simulation, vol. 30, no. 1-3, pp. 360-361, 2016.

[4] O. Naifar, A. B. Makhlouf, and M. A. Hammami, "Comments on "Mittag-Leffler stability of fractional order nonlinear dynamic systems [Automatica 45(8) (2009) 1965-1969]"” Automatica, vol. 75, p. 329, 2017.

[5] A. Ben Makhlouf, M. A. Hammami, and K. Sioud, "Stability of fractional order nonlinear systems depending on a parameter," Bulletin of the Korean Mathematical Society, vol. 54, no. 4, pp. 1321-1309, 2017.

[6] A. Souahi, O. Naifar, A. B. Makhlouf, and M. A. Hammami, "Discussion on Barbalat Lemma extensions for conformable fractional integrals," International Journal of Control, vol. 92, no. 2, pp. 234-241, 2019.

[7] O. Naifar, A. M. Nagy, A. B. Makhlouf, M. Kharrat, and M. A. Hammami, "Finite-time stability of linear fractionalorder time-delay systems," International Journal of Robust and Nonlinear Control, vol. 29, no. 1, pp. 180-187, 2019.

[8] A. Ben Makhlouf, O. Naifar, M. A. Hammami, and B.-w. Wu, "FTS and FTB of conformable fractional order linear systems," Mathematical Problems in Engineering, vol. 2018, Article ID 2572986, 5 pages, 2018.

[9] A. Jmal, A. Ben Makhlouf, A. M. Nagy, and O. Naifar, "Finitetime stability for Caputo-Katugampola fractional-order timedelayed neural networks," Neural Processing Letters, vol. 50, no. 1, p. 607, 2019.

[10] O. Naifar, A. Ben Makhlouf, M. A. Hammami, and L. Chen, "Global practical Mittag Leffler stabilization by output feedback for a class of nonlinear fractional-order systems," Asian Journal of Control, vol. 20, no. 1, pp. 599-607, 2017.

[11] A. Jmal, O. Naifar, A. Ben Makhlouf, N. Derbel, and M. A. Hammami, "On observer design for nonlinear Caputo fractional-order systems," Asian Journal of Control, vol. 20, no. 4, pp. 1533-1540, 2017.

[12] A. Jmal, O. Naifar, A. Ben Makhlouf, N. Derbel, and M. A. Hammami, "Sensor fault estimation for fractionalorder descriptor one-sided Lipschitz systems," Nonlinear Dynamics, vol. 91, no. 3, pp. 1713-1722, 2017.
[13] A. Jmal, O. Naifar, A. Ben Makhlouf, N. Derbel, and M. A. Hammami, "Robust sensor fault estimation for fractional-order systems with monotone nonlinearities," Nonlinear Dynamics, vol. 90, no. 4, pp. 2673-2685, 2017.

[14] A. Jmal, M. Elloumi, O. Naifar, A. Ben Makhlouf, and M. A. Hammami, "State estimation for nonlinear conformable fractional-order systems: a healthy operating case and a faulty operating case," Asian Journal of Control, vol. 22, no. 5, p. 1870, 2019.

[15] H. Wu, X. Zhang, S. Xue, and P. Niu, "Quasi-uniform stability of Caputo-type fractional-order neural networks with mixed delay," International Journal of Machine Learning and Cybernetics, vol. 8, no. 5, pp. 1501-1511, 2017.

[16] G. Rajchakit, P. Chanthorn, M. Niezabitowski, R. Raja, D. Baleanu, and A. Pratap, "Impulsive effects on stability and passivity analysis of memristor-based fractional-order competitive neural networks," Neurocomputing, vol. 417, pp. 290-301, 2020.

[17] G. Rajchakit, P. Chanthorn, P. Kaewmesri, R. Sriraman, and C. P. Lim, "Global Mittag-Leffler stability and stabilization analysis of fractional-order quaternion-valued memristive neural networks," Mathematics, vol. 8, no. 3, p. 422, 2020.

[18] G. Rajchakit, A. Pratap, R. Raja, J. Cao, J. Alzabut, and C. Huang, "Hybrid control scheme for projective lag synchronization of Riemann-Liouville sense fractional order memristive BAM neural networks with mixed delays," Mathematics, vol. 7, no. 8, p. 759, 2019.

[19] R. Sriraman, G. Rajchakit, C. P. Lim, P. Chanthorn, and R. Samidurai, "Discrete-time stochastic quaternion-valued neural networks with time delays: an asymptotic stability analysis," Symmetry, vol. 12, no. 6, p. 936, 2020.

[20] P. Chanthorn, G. Rajchakit, U. Humphries, P. Kaewmesri, R. Sriraman, and C. P. Lim, "A delay-dividing approach to robust stability of uncertain stochastic complex-valued Hopfield delayed neural networks," Symmetry, vol. 12, no. 5, p. 683, 2020.

[21] P. Chanthorn, G. Rajchakit, J. Thipcha et al., "Robust stability of complex-valued stochastic neural networks with timevarying delays and parameter uncertainties," Mathematics, vol. 8 , no. 5 , p. $742,2020$.

[22] A. A. Kilbas, H. M. Srivastava, and J. J. Trujillo, Theory and Application of Fractional Differential Equations, Elsevier, New York, NY, USA, 2006.

[23] I. Podlubny, Fractional Differential Equations, Academic Press, San Diego, CA, USA, 1999.

[24] S. Hussain, H. Sadia, and S. Aslam, "Some generalized Gronwall-Bellman-Bihari type integral inequalities with application to fractional stochastic differential equation," Filomat, vol. 33, no. 3, pp. 815-824, 2019. 